posterior and medio - lateral plane. Joint position sense and postural stability were assessed three times: during rest, following the fatigue protocol, and following the taping. The subjects were received a clinically-used fatigue protocol on a cycle ergometer. The Modified Borg's Rate of Perceived Exertion Scale has been used for fatigue determination.

Results: Joint position sense and postural stability were significantly decreased following fatigue compared to the condition during rest $(p<0.05)$. However, no significant difference was found in terms of joint position sense and postural stability after taping compared to the condition following fatigue $(p>0.05)$.

Conclusions: The hypothesis of this study, that KT could partially compensate for the proprioceptive and balance-related deficits induced by muscle fatigue, was not supported. According to the results of our study, we concluded that the subjects do not benefit from the use of KT for compensating joint position sense and postural stability in condition following fatigue.

References:

[1] Yeung SS, Yeung EW, Sakunkaruna Y, et al. Acute effects of kinesio taping on knee extensor peak torque and electromyographic activity after exhaustive isometric knee extension in healthy young adults. Clin J Sport Med. 2015 May;25(3):284-90.

[2] Halseth T, McChesney JW, DeBeliso M, et al. The effects of Kinesio taping on proprioception at the ankle. J Sports Sci Med. 2004;3:1-7.

[3] Chang HY, Chou KY, Lin JJ, et al. Immediate effect of forearm Kinesio taping on maximal grip strength and force sense in healthy collegiate athletes. Phys Ther Sport. 2010;11:122-127.

Disclosure of Interest: None declared

DOI: 10.1136/annrheumdis-2017-eular.5688

\section{AB1206-HPR ADAPTATION INTO SPANISH OF THE SCLERODERMA HEALTH ASSESMENT QUESTIONNAIRE (S-HAQ)}

P. Quevedo ${ }^{1}$, M.A. Rivero ${ }^{1}$, N. Perez ${ }^{2}$, H. Laborde ${ }^{2}$, G. Earsman ${ }^{1}$, M. Mayer ${ }^{1}$ S. Papasidero ${ }^{3}$, S. Julia ${ }^{3}$, C. Zaffarana ${ }^{4}$, R. Maldonado ${ }^{5}$, M. Khoury $^{6}$, M. Lara ${ }^{7}$ C. Castell ${ }^{7}$, A. Curti ${ }^{7}$, J.C. Barreira ${ }^{1}$, V. Steen ${ }^{8} .{ }^{1}$ British Hospital; ${ }^{2}$ Hospital de Clinicas José de San Martin; ${ }^{3}$ Tornu Hospital; ${ }^{4}$ Psychophysical Rehabilitation Institute (Irep), Buenos Aires; ${ }^{5}$ Padilla Hospital, TUCUMAN; ${ }^{6}$ Statistics, British Hospital, Buenos Aires; ${ }^{7}$ Luis Lagomaggiore Hospital, MENDOZA, Argentina;

${ }^{8}$ Georgetown University, Washington, United States

Background: The Health Assessment Questionnaire (HAQ) is an instrument administered to patients to self-report functional status originally in rheumatoid arthritis (RA). In Argentina, it has been translated and validated for RA in 2004. For diffuse SSc, HAQ has been associated to morbidity and mortality.

Objectives: To adapt S-HAQ into Spanish and to assess its validity in SSc patients in Argentina.

Methods: S-HAQ was translated following a forward-backward translation procedure of the original English version, and transcultural adaptation was performed by a comprehension test reaching the final Spanish version. SSc patients that fulfilled ACR 80 criteria and early Systemic Sclerosis according to Le Roy and Medsger criteria were included. Patients with overlap were excluded. Cronbach's alpha and item-item item-total correlations were used to assess internal consistency. Construction validity was analyzed through factor analysis with Varimax rotation. Continuous variables were compared by t-test, Mann-Whitney or Kruskal-Wallis test, and categorical variables by chi-square or Fisher's test. A value of $p<0.05$ was considered significant.

Results: I9An adapted Argentine-Spanish version of S-HAQ was developed. One hundred patients were surveyed; $84 \%$ were female, mean age $54 \pm 12.8$ years and disease duration $8.8 \pm 9.1$ years. Limited SSc was more frequent (63\%), followed by diffuse SSc (36\%). Serologically, $89 \%$ were ANA positive, $27 \%$ had anti Scl 70 and $41 \%$ had anti centromere antibodies. Median Rodnan score (mRSS) was $9.8(0-40.5)$ and median activity measured by EUSTAR was $1.25(0-6)$. Median S-HAQ was $0.62(0-2.5)$, Cronbach's alpha 0.89 , and when removing questions one by one the coefficient decreased. Median VAS (visual analogue scale) was $0.57(0-2.8)$. Factor analysis identified two factors for the S-HAQ: factor 1 : dressing (0.61), arising (0.68), reach $(0.63)$, and personal hygiene $(0.70)$; factor 2: eating (0.68), grip (0.72), walking (0.49), usual activities (0.62). For questions, three factors were identified through VAS: factor 1 : overall disease severity (0.63) and gastro-intestinal symptoms (0.57); factor 2: Raynaud's (0.66), digital ulcers (0.56); factor 3 : respiratory symptoms $(0.43)$. There was a statistically significant association between higher values of S-HAQ and higher values of mRSS $(1.1 \pm 0.74$ vs. $0.64 \pm 0.5 p=0.002)$ and also with seropositivity for anti-Scl 70 $(p=0.003)$. Higher values of total VAS were associated to female gender $(0.75 \pm 0.5$ vs. $0.49 \pm 0.71, p=0.01)$. There was a significant association between $S-H A Q$ and MEDSGER $(p=0.04)$ and EUSTAR $(p=0.03)$ scores; likewise, between VAS and MEDSGER $(p=0.00)$ and EUSTAR $(p=0.00)$ scores.

Conclusions: A Spanish version of S-HAQ was developed, showing an acceptable reliability and validity.

Disclosure of Interest: None declared

DOI: 10.1136/annrheumdis-2017-eular.4025

\section{AB1207-HPR PATIENT SATISFACTION IN A RHEUMATOID ARTHRITIS SPECIALIZED CENTER}

$\underline{\text { S. Henao }}^{1}$, F. Rodriguez ${ }^{2}$, P. Santos-Moreno ${ }^{3}$, L. Villarreal ${ }^{4}$, M. Cabrera ${ }^{5}$, A. Malpica ${ }^{6}$, D. Buitrago-Garcia ${ }^{6} .{ }^{1}$ Patient service, ${ }^{2}$ Patient Program;

${ }^{3}$ Rheumatology; ${ }^{4}$ Psychology and processes; ${ }^{5}$ Engineer and processes; ${ }^{6}$ Epidemiology, Biomab, Center for Rheumatoid Arthritis, Bogota, Bogota, Colombia

Background: According to the Beryl Institute's, patient experience (PX) is "the sum of all interactions, shaped by an organization's culture, that influence patient perceptions, across the continuum of care"; nowadays patient satisfaction is considered as one of the quality for performance in health systems (1). In order to provide a multidisciplinary quality care to patients with RA in centers of excellence (CoEs) under the coordination of a rheumatologist, provide comprehensive management of patients with this pathology, ensuring approachability to medical appointments and treatment, in order to get better clinical outcomes and improve patient safety and satisfaction of the health services provided.

Objectives: To measure levels of satisfaction of RA patients treated at a specialized center and to evaluate patient service.

Methods: In a RA specialized center during a 24 month period we performed a satisfaction survey in order to evaluate the health services provided. We evaluated the timing on attention, appointment assignment, information provided, the treatment received by the healthcare team, facilities among others. Patients evaluated the services provided in a scale from 1 to 4 , were 1 was very bad, 2 regular, 3 good and 4 excellent. Descriptive epidemiology was performed for each variable presented.

Results: We collected 1125 surveys during 2015 and 2016, 45\% considered to have a timely care, the mean of waiting time for an appointment was $9 \min \pm 8$; regarding the appointment assignment $96 \%$ of the patients evaluated it as good or excellent (mean $3.5 \pm 0.7$ ), $80 \%$ considered that the information provided was clear and useful, $90 \%$ reported to receive a kind and friendly treatment and to considered the facilities as good or excellent. When we evaluated the satisfaction regarding the health care team $50 \%$ of patients evaluated the rheumatologist, nurse, nutritionist, physical therapist, psychologist and physiatrist as good and $40 \%$ as excellent.

Conclusions: Although we found that our patients are highly satisfied, there is a large opportunity to improve our services. Also, this evidence can support further research projects in order to increase the patient's satisfaction.

References:

[1] Th VP. Creating the ideal patient experience. Journal of medicine and life. 2016:9(4):380-385.

Disclosure of Interest: None declared

DOI: 10.1136/annrheumdis-2017-eular.5380

\section{HPR epidemiology and public health (including prevention)}

\section{AB1208-HPR PSORIASIS INDUCED BY TNF ANTAGONIST THERAPY. ANALYSIS OF 13 CASES}

À. Martinez-Ferrer ${ }^{1}$, A. Mateu Puchades ${ }^{2}$, C. Vergara Dangond ${ }^{1}$, M. Aguliar Zamora $^{1}$, L. Montolio ${ }^{1}$, S. Santos ${ }^{2}$, È. Valls Pascual ${ }^{1}$, D. Ybáñez García ${ }^{1}$, J.J. Alegre Sancho ${ }^{1} .{ }^{1}$ Rheumatology; ${ }^{2}$ Dermatology, Hospital Dr Peset, Valencia, Spain

Background: Tumor necrosis factor (TNF) antagonist drugs have been shown to be effective in different inflammatory arthropathies and autoimmune pathologies, including psoriasis. However, an unexpected side effect has been observed: the new occurrence or worsening of psoriatic lesions.

Objectives: The aim of this study is to describe the cases of induction or worsening of psoriasis in patients treated with TNF antagonist therapy in our center.

Methods: Retrospective observational study, review of cases of new or worsening psoriasis in patients with TNF antagonist at the University Hospital Dr. Peset from October 2008 to November 2016. A total of 13 cases were obtained.

Results: Thirteen patients, 8 females and 5 males with mean age 46 years $( \pm$ 16). $38 \%$ of patients received treatment for Crohn's disease, $31 \%$ for rheumatoid arthritis (RA), $31 \%$ for psoriatic arthropathy (APs), $8 \%$ for ankylosing spondylitis $(\mathrm{AE})$ and another $8 \%$ for psoriasis. Two patients were diagnosed of more than one pathology: Crohn's disease associated with APs and Crohn's disease associated with RA. Sixty one percent had no known personal history of psoriasis, in one of them the family history of psoriasis was recorded. Infliximab was used in $38.5 \%$ of cases, followed by adalimumab and golimumab in $23 \%$ each and etanercept in $15.4 \%$. The mean latency time since drug introduction was 9.3 months (2-26). There were 12 cases of psoriasis and 1 case of pityriasis lichenoides (histologically confirmed). Lesion morphology included pustular psoriasis in $91 \%$, scalp psoriasis in $25 \%$, guttate lesions in $25 \%$, plaque psoriasis in $8 \%$, and inverse psoriasis in $8 \% ; 58 \%$ experienced lesions of more than one type. There were no cases of nail, mucosal or erythrodermic psoriasis. The psoriasiform lesions resolved without interruption of TNF antagonist therapy in $53.85 \%$. Of the 6 patients who required discontinuation, 3 patients were switched to another anti-TNF drug (adalimumab, 
golimumab and certolizumab) and all 3 had recurrence of the lesions, in 2 patients the anti-TNF was replaced by a non-anti-TNF biological. Topical treatment was used in all cases, one patient also required systemic treatment with methotrexate. Conclusions: TNF antagonist induced psoriasis is a well-described adverse event. Pustular psoriasis is the most frequent presentation. In most cases there is no personal or family history of psoriasis. Topical therapy may be effective but some patients require discontinuation of the drug. Skin lesions can reappear when switching to another anti-TNF drug.

Disclosure of Interest: None declared

DOI: 10.1136/annrheumdis-2017-eular.2877

\section{AB1209-HPR THE PREVALENCE OF DENTAL AND SINUS INFECTION IN PATIENTS WITH RHEUMATOID ARTHRITIS BEFORE BIOLOGIC THERAPY INITIATION: USEFULNESS OF A SYSTEMATIC SCREENING?}

C. Tawil, E. Descamps, M. Forien, A. Gardette, E. Palazzo, S. Ottaviani, P. Dieudé. Rheumatology Department, Bichat Hospital, Paris, France

Background: Introduction of the biologic therapies has dramatically improved the outcome of severe rheumatoid arthritis (RA). Biologic therapies play a central role in the control of synovial inflammation. However they also decrease host defenses leading to an increased rate of infection. Because of their adverse effects, a careful assessment is needed before their initiation. A systematic assessment of dental or sinus infection before a biologic therapy is not required.

Objectives: The aim of our study was to assess the prevalence and the usefulness of a systematic screening of oral (dental and/or sinus) infection of RA patients before biologic therapy initiation.

Methods: This was a monocentric retrospective study. We included RA (ACR/EULAR 2010 criteria) patients with active disease despite disease-modifying anti-rheumatic drugs (DMARDs) and requiring biologic therapy initiation between 2010 and 2016. The following parameters were collected: demographic and disease characteristics, disease activity (C-reactive protein, disease activity score (DAS) 28), currents therapies (DMARDS, corticosteroids). Dental infection was assessed by stomatologist after clinical and panoramic dental X-ray evaluation. Sinusitis was defined on sinus computed tomography as partial or complete opacification of one or more sinus cavities. Factors associated with oral infections were analyzed in uni- and multivariate models.

Results: We included 223 RA patients $(79.4 \%$ of female, mean \pm SD disease duration of $8.9 \pm 8.6$ years). The mean age was $54 \pm 10.9$ years, $70.8 \%$ rheumatoid factor (RF) positive, $84.4 \%$ anti-citrullinated protein antibody (ACPA) positive and $68.1 \%$ had radiographic damages. The mean DAS 28 was $5.5 \pm 2.6 ; 71 \%$ of patients received corticosteroids (mean $7 \mathrm{mg}$ per day of equivalent prednisone) and $63 \%$ methotrexate (mean $17.8 \mathrm{mg}$ per week). No patient had pain or other sinus or dental symptoms. Before biologic agent initiation, systematic dental and sinus screening revealed an oral infection in $31.5 \%$ of patients (dental: $20.2 \%$ and sinus: $14.8 \%$ ). In univariate analysis, active smoking was associated with a higher risk of oral infection $(\mathrm{OR}=2.16[1.02-4.57], \mathrm{p}=0.038)$ and methotrexate with $a$ lower rate $(O R=0.43$ [0.23-0.81], $p=0.006)$. Corticosteroid, disease duration, DAS 28, RF, ACPA and structural damages were not associated with oral infection. No significant association was confirmed with oral infection using multivariate analysis.

Conclusions: In our study, one third of RA patients requiring biologic agents had asymptomatic oral infection. The high prevalence of oral infection in RA patients suggests the usefulness of systematic dental and sinus screening before biologic therapy initiation.

Disclosure of Interest: None declared

DOI: 10.1136/annrheumdis-2017-eular.4037

\section{AB1210-HPR MENDELIAN RANDOMIZATION ANALYSIS INDICATES SERUM URATE HAS A CONDITIONAL CAUSAL EFFECT ON SERUM CREATININE AND RENAL FUNCTION}

J. Liu ${ }^{1}$, H. Zhang ${ }^{1}$, Z. Dong ${ }^{1}$, J. Zhou ${ }^{1}$, Y. Ma ${ }^{1}$, Y. Li ${ }^{1}$, Q. Qian ${ }^{1}$, Y. Yang ${ }^{1,2}$, X. Wang ${ }^{1,2}$, H. Zou ${ }^{3,4}$, L. Jin ${ }^{1,2}$, J. Wang ${ }^{1,2,4}$. ${ }^{1}$ State Key Laboratory of Genetic Engineering, Collaborative Innovation Center for Genetics and Development, School of Life Sciences, Fudan university, Shanghai; ${ }^{2}$ Fudan-Taizhou Institute of Health Sciences, Taizhou; ${ }^{3}$ Division of Rheumatology, Huashan Hospital, Fudan University; ${ }^{4}$ Institute of Rheumatology, Immunology and Allergy, Fudan university, Shanghai, China

Background: Uric acid, the weak organic acid and the end product of purine nucleotide degradation, is excreted predominantly by the proximal tubules [1]. Although large numbers of epidemiological, molecular and animal studies have focused on various pathogenic effects of serum uric acid, inclding chronic kidney disease (CKD), metabolic syndrome, and coronary artery disease [2], whether the serum uric acid is an independent risk factor or has causal impact on serum creatinine ( $\mathrm{SCr}$ ) and renal function remains unclear.

Objectives: We aim to study the effect of serum uric acid on renal function by applying the method of Mendelian randomization.

Methods: The study was represented by estimated glomerular filtration rate (eGFR) with potential confounding factors in 3,734 Chinese subjects. Four genetic variants of uric acid transporter genes (rs1481012 [ABCG2], rs16890979
[SLC2A9], rs2231137 [ABCG2] and rs3799352 (SLC17A1]) were selected for this study as they had highest correlation with serum uric acid in Chinese population in our previous study. In this research, serum uric acid was selected as exposure, genetic risk score of uric acid transporters was selected as instrumental variable, and SCr and eGFR were selected as the outcomes.

Results: 1) The results of the analysis showed that increased serum uric acid has a causal effect on reducing estimated glomerular filtration rate in both female population and the subjects who were under 65 years old. Because of protective effects on renal function of ovarian hormones such as estrogen, we postulated that estrogen might be the cause leading to the difference between men and women. 2) We also found that increased serum uric acid led to the damage of renal function in the subjects with normal eGFR value. 3) In addition, the serum uric acid was a risk factor to renal function in the subjects with relative high level of fasting glucose or who were smoking currently. Because of metabolic defects in people affected by diabetes, renal glucose reabsorption was increased, thus further sustaining hyperglycemia in patients.

Conclusions: Serum urate has causal effects on renal dysfunction in either female or individuals of under 65 , or normal eGFR, or high level of fasting glucose, or current smokers.

References:

[1] Pasalic D, Marinkovic N, Feher-Turkovic L. Biochem Medica 2012;22(1):63-75. [2] Sakhaee K. J Nephrol 2014;27(3):241-5.

Acknowledgements: National Natural Science Foundation of China (31521003), Science and Technology Committee of Shanghai Municipality (11DJ1400102), International S\&T Cooperation Program of China (2013DFA30870), Ministry of Science and Technology (2011BAI09B00), 111 Project (B13016), and Program for 2012 Outstanding Medical Academic Leader for Hejian Zou. Computational support was provided by the High-End Computing Center located at Fudan University.

Disclosure of Interest: None declared

DOI: 10.1136/annrheumdis-2017-eular.5601

\section{AB1211-HPR THE USE OF SUBCUTANEOUS METHOTREXATE IN POLISH PATIENTS WITH RHEUMATOID ARTHRITIS}

M. Przygodzka ${ }^{1}$, K. Sikorska-Siudek ${ }^{1}$, R. Radomski ${ }^{2}$, S. Bojanowski ${ }^{2}$. ${ }^{1}$ Mazovian Centre of Rheumatology and Osteoporosis; ${ }^{2}$ iEHR.eu, Warsaw, Poland

Background: Methotrexate (Mtx) should be the drug of the first choice in rheumatoid arthritis (RA) if there are no contraindications to use it. The efficacy of $\mathrm{Mtx}$ is measured by remission or low disease activity and depends on the dose taken. Higher doses (25-30 mg/week) are more effective, but intolerance is the main cause of discontinuation of oral treatment. Subcutaneous Mtx is efficient alternative in those cases.

Objectives: The aim of this study was to evaluate subcutaneous Mtx use frequency in Polish patients and change oral form for subcutaneous as well.

Methods: The disease activity was assessed by Disease Activity Score 28 (DAS 28 ) during the first visit (V1) and after 3 months therapy (V2) and compliance with therapy as well.

Results: There were 194 RA patients diagnosed by the ACR and 1997 and/or ACR/EULAR 2010 criteria. 144 patients were treated by oral Mtx (group A) and 50 patients (group B) by subcutaneous Mtx at the time of study enrolment (V1).37 patients of group $A(26 \%)$ required changes in therapy during V2, $24(17 \%)$ were switched to subcutaneous Mtx (group A1). 6 patients of group B (12\%) required change of treatment during V2, including 2 patients (4\%) with subcutaneous Mtx, who were switched to oral Mtx.The main cause of changing therapy from oral to subcutaneous was gastrointestinal intolerance of high dose of Mtx. 69 patients $(12 \%)$ of group A required additional steroid therapy compared to $18(36 \%)$ of group B. Average DAS 28 decreased by 0.58 in group A1 in oral treatment and during the subcutaneous treatment time decreased by next 0.23 . In group A1 during oral treatment 14 (58\%) patients used $25 \mathrm{mg} /$ week and 20 patients (83\%) used $25 \mathrm{mg} /$ week during subcutaneous treatment time.

Conclusions: Patients treatment by oral Mtx often require modification of therapy in comparison to patients treated by subcutaneous Mtx, including more frequent use of steroids

The main cause of oral intolerance are ailments of the digestive system.

Change of oral to subcutaneous therapy allows administration of higher doses of Mtx and results in decrease of DAS28 in comparison to the patients continuing their oral treatment.

Disclosure of Interest: None declared

DOI: 10.1136/annrheumdis-2017-eular.6963

\section{AB1212-HPR DYNAMICS OF ARTICULAR SYNDROME IN RHEUMATOID ARTHRITIS AGAINST CORRECTION OF PROGESTERONE INSUFFICIENCY}

M. Salokhiddinov, A. Ahmedov. Rheumatology and Osteopathy, Tashkent Medical Academy, Tashkent, Uzbekistan

Background: According to the literature, sex hormones manifest themselves as immune modulators that inhibit the ones and stimulate other immune functions. Studies have shown that patients with RA, decrease of estradiol and particularly 